\title{
Multiuser Detection for CDMA Systems
}

\author{
A. Duel-Hallen \\ J. Holtzman \\ Z. Zvonar
}

Center for Communications and Signal Processing Department of Electrical and Computer Engineering North Carolina State University

TR-94/21
October 1994 
lilagotive

\title{
MULTIUSER DETECTION FOR CDMA SYSTEMS
}

\author{
Alexandra Duel-Hallen*, Jack Holtzman**, Zoran Zvonar*** \\ ${ }^{*}$ CCSP, North Carolina State University, ${ }^{* *}$ WINLAB, Rutgers University, ${ }^{* * *}$ Woods Hole \\ Oceanographic Institute
}

\section{$\underline{\text { Abstract }}$}

Present day direct sequence code division multiple access (DS/CDMA) systems treat each user separately as a signal, with all of the other users representing interference or noise. Potentially significant capacity improvements can be achieved if the negative effect that each user has on the others is cancelled. In other words, joint detection (multiuser detection), rather than separate detection, is beneficial. Optimal multiuser detection is, however, too complex. Thus, suboptimal approaches have been investigated. The basic principles of optimal and suboptimal multiuser detection are well understood now and effort is underway on investigating practical and economic feasibility.

\section{Introduction}

Spread spectrum has been very successfully used by the military for decades. Recently, spread spectrum based code division multiple access (CDMA), has taken on a significant role in cellular and personal communications. Multiple access allows multiple users to share limited resources such as frequency (bandwidth) and time. There are a number of multiple access schemes including more than one type of CDMA. We shall concentrate on one type, Direct Sequence CDMA (DS/CDMA). CDMA has been found to be attractive because of such characteristics as potential capacity increases over competing multiple access methods, anti-multipath capabilities, soft capacity and soft handoff.

We shall not here cover all the background of DS/CDMA since that has been well explained in recent literature (see e.g., [VI94]). In fact, the present article may be viewed as a supplement to that literature with an update on some potential enhancements to the versions of DS/CDMA currently being developed (see [GI91], [PI91])*. We will show that there is a natural modification of the present systems, multiuser detection, that is potentially capable of significant capacity increases. By "natural modification" we mean a modification that can be made conceptually clear, not that it is easy to implement. Indeed, the optimal multiuser detector is much too complex and most of the present research addresses the problem of simplifying multiuser detection for implementation. The objective of this article is to make the basic idea intuitive and then show how investigators are trying to reduce the idea to practice. As a result of these investigations, an answer is expected to the following question: Is there a suboptimal multiuser detector that is cost effective to build with significant enough performance advantage over present day systems?

We will first review some salient features of CDMA systems needed for the discussion following.

* A comprehensive reference set on spread spectrum until 1985 is [SI85]. 


\subsection{Limitations of a Conventional CDMA System}

A conventional DS/CDMA system treats each user separately as a signal, with the other users considered as interference (multiple access interference, MAI), or noise. The detection of the desired signal is protected against the interference due to the other users by the inherent interference suppression capability of CDMA, measured by the processing gain. The interference suppression capability is, however, not unlimited and as the number of interfering users increases, the equivalent noise results in degradation of performance, i.e., increasing bit error rate (BER). Even if the number of users is not too large, some users may be received at such high signal levels that the desired user may be swamped out. This is the near/far effect: users near the receiver are received at higher powers than those far away, and those further away suffer a degradation in performance. Even if users are at the same distance, there can be an effective near/far effect because some users may be received during a deep fade. DS/CDMA systems are very sensitive to the near/far effect and the recent success of DS/CDMA has, in large part, been due to the successful implementation of relatively tight power control, with attendant added complexity.

There are thus two key limits to present DS/CDMA systems:

- All users interfere with all other users and the interferences add to cause performance degradation.

- The near/far problem is serious and tight power control, with attendant complexity, is needed to combat it.

\subsection{Multipath Propagation}

One other aspect of CDMA that we need to review is the ability to combat multipath reception of signals [TU84]. Due to multiple reflections, the received signal contains delayed, distorted replicas of the original transmitted signal. First, consider what happens in a non-spread spectrum system. When the multiple reflections, called multipath signals or simply multipaths, from one transmitted bit are received within the time duration of one bit, the received signal consists of the superposition of the several signal replicas, each with its own amplitude and phase. It is important to recognize that this superposition is the addition of complex quantities. Due to the motion of the mobile (or, even of the base station in some systems), the relative phases of the received signals are continually changing. This results in successive reinforcement and interference of the superposed multipath signals, resulting in very large time variations in the received signal. Such variations are referred to as Rayleigh fading (or Rician fading, if there is a direct component in addition to the reflections). The variations due to Rayleigh fading are a serious cause of performance degradation and a communication system must be designed carefully, taking that into account.

We shall refer to systems where all of the multipath signals arrive within one bit interval as "narrowband". On the other hand, the bit rate may be so high that multipath signals from one bit arrive over a duration longer than that of one bit. Such systems will be called "wideband". The Rayleigh fading effect is less pronounced, because there are fewer multipath signals from one bit arriving during the bit duration. 
CDMA systems are inherently wideband when the chip duration, as opposed to the longer bit duration, is compared to the time between multipath receptions. One can then combat multipath interference by multipath reception, whereby the different multipath arrivals are considered as independent receptions of the signal and are used to give a beneficial time diversity. This is usually done with a RAKE receiver, the name apparently from the operation of a RAKE with a number of teeth pulling in a number of items simultaneously. So, instead of multipath being just a source of performance degradation, the multipaths are used to provide the benefit of diversity [PR89].

\subsection{Interference Cancellation and Multiuser Detection}

In a conventional CDMA system, all users interfere with each other. Potentially significant capacity increases and near/far resistance can theoretically be achieved if the negative effect that each user has on others can be canceled. A more fundamental view of this is multiuser detection in which all users are considered as signals for each other. Then, instead of users interfering with each other, they are all being used for their mutual benefit by joint detection. The drawback of optimal multiuser detection is one of complexity so that suboptimal approaches are being sought. There is a wide range of possible performance/complexity combinations possible. Much of the present research is aimed at finding an appropriate tradeoff between complexity and performance.

\subsection{Multiuser Detection in Cellular Systems}

In a cellular system, a number of mobiles communicate with one base station (BS). Each mobile is concerned only with its own signal while the BS must detect all the signals. Thus, the mobile has the information only about its own chip sequence while the base station has the knowledge of all the chip sequences. For this reason, as well as less complexity being tolerated at the mobile (where size and weight are critical), mulituser detection is currently being envisioned mainly for the BS, or in the reverse link (mobile to BS). It is important to realize, however, that the BS maintains information only on those mobiles in its own cell. This plays a role in the limitations on improvements to be expected in a multiuser detection system, to be discussed next.

\subsection{Limitations to Improvements}

Before we discuss multiuser improvements to the conventional DS/CDMA detector, it is important to define factors that limit such improvement [HO94]. One factor is intercell interference in a system that cancels only the intracell interference ${ }^{* *} I$. For intercell interference which is a fraction $f$ of the the intracell interference, the bound of capacity increase (all of the intracell interference is cancelled) is $(1+f) / f$. For $f=0.55$, this factor is 2.8 [VI94]. Observe that with a sectorized antenna, it is conceivable to cancel users from another sector and thus improve the bound.

\footnotetext{
** Intracell interference is from interferers in the same cell as the desired user while intercell interference is from interferers outside the cell. It is conceivable to try to cancel intercell interference but this presents another level of difficulty.
} 
Another limiting factor is the fraction, $f_{c}$, of energy captured by a RAKE receiver. That is, a RAKE receiver with $L$ branches or "fingers" will try to capture the power in the $L$ strongest multipath rays but there will be additional received power in additional rays. For the conventional detector, this is selfinterference. Ref. [CH92] gives examples of the fraction of captured power. The fraction of captured power is a function of chip rate and delay spread as well as the number of RAKE branches. So, combining the two effects (measured by $f$ and $f_{c}$ ), the total interference before cancellation is $(1+f) I$ (neglecting the smaller self-interference due to uncaptured multipath power of the desired user). Cancellation removes at most $f_{c} I$ so the bound on improvement is $(1+f) /\left(1+f-f_{c}\right)$. For $f_{c} \approx 1$, the above bound of 2.8 on capacity improvement remains. For $f_{c}=0.5$, the bound is reduced to 1.5 .

It should be recognized that multiuser detection is used not only to increase capacity but also to alleviate the near/far problem, and the preceding bound does not account for that benefit. Relaxing the power control requirement actually translates into a capacity benefit which is, however, more difficult to quantify than by the above simple signal/interference argument. A multiuser detector could recapture part of this reduction by reducing variability (or relax the requirements on power control).

To put these constraints on improvements into further perspective, we are assuming here that multiuser detection is a candidate primarily for the reverse link for reasons given earlier. Since the reverse link is usually more limiting than the forward link, increasing the reverse link capacity will improve the overall system capacity. But increasing it beyond the forward link capacity will not further increase the overall system capacity. Thus,

(i) The potential capacity improvements in cellular systems are not enormous (order of magnitude) but certainly non-trivial

(ii) Enormous capacity improvements only on the reverse link (the candidate for multiuser detection) would only be partly used anyway in determining overall system capacity

(iii) Hence, the cost of doing multiuser detection must be as low as possible so that there is a performance/cost tradeoff advantage to multiuser detection.

The bottom line is that there are significant advantages to multiuser detection which are, however, bounded and a simple implementation is needed.

\subsection{Historical Background}

The idea of interference cancellation arises in many contexts, e.g., noise cancellation in speech [LI83] and adaptive interference canceling as in Chapter 12 of [WI85]. There are thus a number of non-CDMA references with ideas similar to those being currently studied for CDMA. We should distinguish between canceling noise which has no useful purpose (as in [LI83], Chap. 12 of [WI85]) from canceling interference which is due to other signals which are themselves to be detected. A couple of non-CDMA examples in the latter category are [SA85] and [CA87]. The CDMA case considered here is of the second type, where the signals being canceled are of interest also. It should be remarked, however, that the first 
type of cancellation also is of importance in CDMA systems, e.g., in suppressing narrowband interference (this is not discussed in this article, but is discussed in Section 5 of [PI91]). Both types of interference cancellation have in common the goal of removing from a desired signal a noise-like interference. But in the second type (the type considered here), the fact that the signals being removed are themselves information carrying leads to a new viewpoint, one of simultaneously detecting all the information carrying signals.

The first CDMA interference cancellation references we are aware of are [SC79] and [KO83]. Both of these papers delineate a number of ideas that are present in much of the ongoing research. Estimates based on mean square error and maximum likelihood are discussed in [SC79]. Reference [KO83] shows how cancellation is implemented by solving simultaneous equations, in essence, by inverting a key matrix. There were subsequently a number of papers with variants of the ideas of [SC79] and [KO83]. Significant theoretical steps forward were taken in [VE86], [VE86a] (with earlier references), in analyzing the structure and complexity of optimal receivers. This work triggered a new research effort on suboptimal algorithms. The strong connection between MAI and intersymbol interference (ISI) was also made in [VE86]. There are aspects of MAI, however, that are not shared by ISI:

- The near/far problem, and

- MAI is affected by the relationship among user chip sequences (codes) as well as by the imperfections of the radio channel, while ISI is due only to the channel.

Thus while equalization (used to combat ISI) will play a role in the multiuser detectors to be discussed, it should not be expected that it can be used without modification.

Recent survey papers include [VE94], [HO94]. We shall let the rest of the references be brought up as needed in the discussion.

\section{Multiuser Detection: Concept and Techniques.}

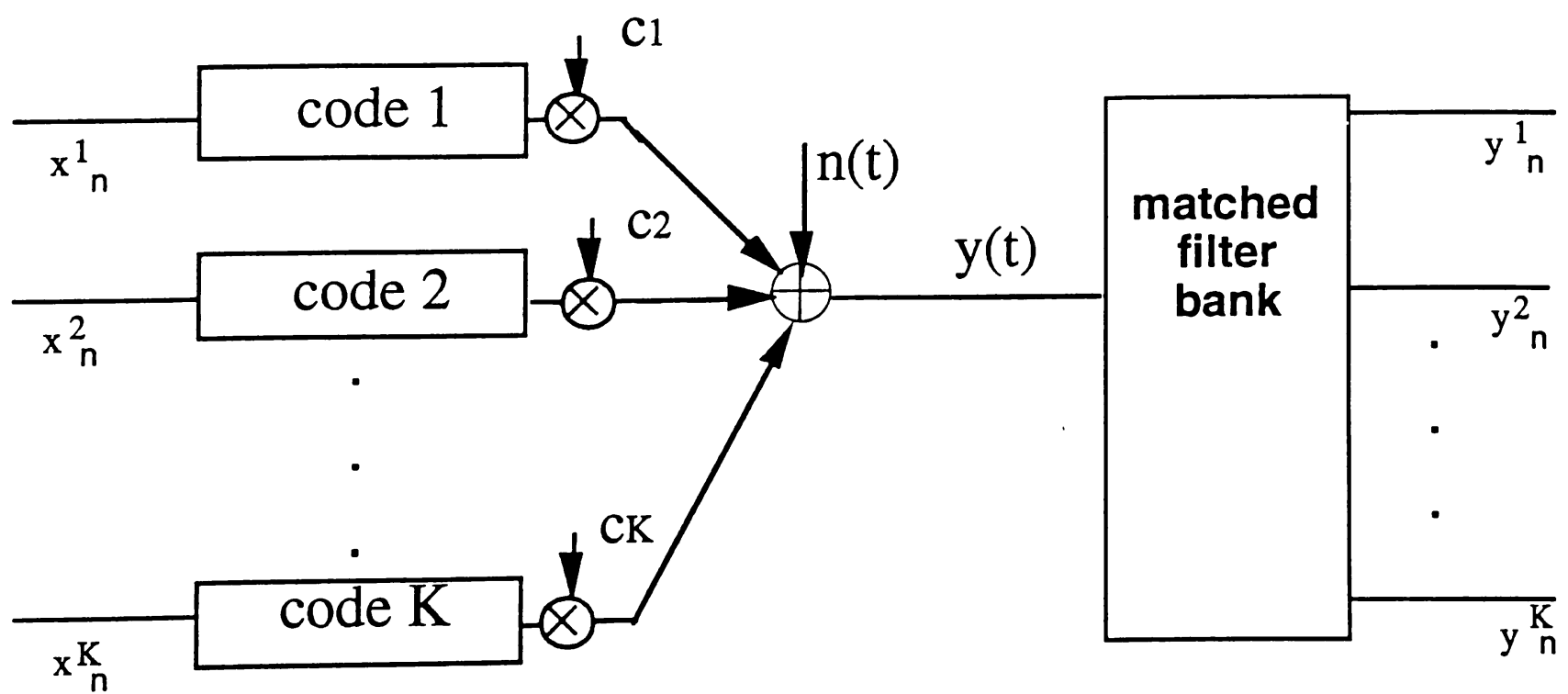

Figure 2-1. The CDMA Channel Model 


\subsection{The CDMA Channel Model and Approaches to Detection.}

A CDMA channel with $\mathrm{K}$ users sharing the same bandwidth is shown in Figure 2-1. The signaling interval of each user is $\mathrm{T}$ seconds, and the input alphabet is antipodal binary: $\{+1,-1\}$. The objective is to detect those polarities, which contain the transmitted information. During the $\mathrm{n}$-th signaling interval, the input vector is $x_{n}=\left(x_{n}{ }^{1}, \cdots, x_{n}{ }^{K}\right)^{T}$, where $x_{n} k^{k}$ is the input symbol of the $k$-th user. User $k(k=1, \ldots, K)$ is assigned a signature waveform (or code, or spreading chip sequence) $s_{k}(t)$ which is zero outside $[0, T]$ and is normalized

$$
\int_{0}^{T} s_{k}(t)^{2} d t=1
$$

Pulse amplitude modulation is employed at the transmitter. The baseband signal of the $\mathrm{k}$-th user is

$$
u_{k}(t)=\sum_{i=0}^{\infty} x_{i} k_{i}{ }_{i}^{k} s_{k}\left(t-i T-\tau_{k}\right)
$$

where $\tau_{\mathrm{k}}$ is the transmission delay, and $\mathrm{c}_{\mathrm{i}} \mathrm{k}$ is the complex channel attenuation. According to (2-1), each user's signal travels along a single path, so this model does not illustrate multipath propagation. The effect of multipath is discussed in sections 1 and 3. For synchronous CDMA, the delay $\tau_{k}=0$ for all users. For asynchronous CDMA, the delays can be different. The channel attenuation is a complex number

$$
c_{i} k=\sqrt{w_{i}^{k}} \exp \left(j \theta_{i} k\right)
$$

where $w_{i} k^{k}$ and $\theta_{i} k$ are the received power and phase of the $k$-th user, respectively. The received signal (at baseband) is the noisy sum of all the users' signals:

$$
y(t)=\sum_{k=1}^{K} u_{k}(t)+z(t),
$$

where $z(t)$ is the complex additive white gaussian noise (AWGN). The first step in the detection process is to pass the received signal $\mathrm{y}(\mathrm{t})$ through a matched filter bank (or a set of correlators). It consists of $\mathrm{K}$ filters matched to individual signature waveforms followed by samplers at instances $T n+\tau_{k}, k=1, \ldots, K, n=1,2$, ... . The outputs of the matched filter bank form a set of sufficient statistics about the input sequence $x_{n}$ given $y(t)$ [VE86]. Thus, we will consider the equivalent discrete-time channel model which arises at the output of the matched filter bank.

For the rest of this section, we will concentrate on a very simplified DS/CDMA system. (There are a number of simplifications which will be exposed in the rest of the article. In fact, each relaxation of 
simplification will represent another factor to consider for the multiuser detection system.) We will consider a synchronous CDMA system with real channel attenuation. The synchronous assumption simplifies analysis and often permits the derivation of closed-form expressions for the desired performance measures. These are useful since similar trends are found in the analysis of the more complex asynchronous case. The real model is convenient for analyzing coherent methods, and can be easily generalized to the complex case. For synchronous CDMA, the output signal $y(t)(2-2)$ for $n T \leq t<(n+1) T$ does not depend on the inputs of other users sent during past or future time intervals. Consequently, it is sufficient to consider a one-shot system with input vector $\mathbf{x}_{n}=\left(x^{1}, \cdots, x^{K}\right)^{T}$, real positive channel attenuations (amplitudes) $\mathrm{c}^{1}=\sqrt{\mathrm{w}^{1}}, \cdots, \mathrm{c}^{\mathrm{K}}=\sqrt{\mathrm{w}^{\mathrm{K}}}$ and real additive white Gaussian noise $\mathrm{z}(\mathrm{t})$ with power spectral density $\mathrm{N}_{0}$. The sampled output of the $\mathrm{k}$-th matched filter (matched to the signature waveform of user $\mathrm{k}$ ) is

$$
\begin{aligned}
y_{k} & =\int_{0}^{T} y(t) s_{k}(t) d t=\int_{0}^{T} s_{k}(t)\left[\sum_{j=1}^{K} s_{j}(t) c^{j} x^{j}+z(t)\right] d t \\
& =c^{k} x^{k}+\sum_{j \neq k}^{K} x^{j} c^{j} \int_{0}^{T} s_{k}(t) s_{j}(t) d t+\int_{0}^{T} s_{k}(t) z(t) d t
\end{aligned}
$$

Note that $\mathrm{y}_{\mathrm{k}}$ consists of three terms. The first is the desired information which gives the sign of the information bit $x_{k}$ (which is exactly what is sought). The second term is the result of the multiple access interference (MAI), and the last is due to the noise. The second term typically dominates the noise so that one would like to remove its influence. Its influence is felt through the cross-correlations between the chip sequences and the powers of users. If one knew the cross-correlations and the powers, then one could attempt to cancel the effect of one user upon another. This is, in fact, the intuitive motivation for interference cancellation schemes.

Suppose there are only two users in the system. Let $\mathrm{r}$ be the cross correlation between the signature waveforms of the two users $r=\int_{0}^{T} s_{1}(t) s_{2}(t) d t$. In this case, the outputs of the matched filters are

$$
y^{1}=c^{1} x^{1}+r c^{2} x^{2}+z^{1} \text { and } y^{2}=c^{2} x^{2}+r c^{1} x^{1}+z^{2}
$$

The MAI terms for users 1 and 2 are $r c^{2} x^{2}$ and $r c^{1} x^{1}$, respectively. If these terms were not present, the single user system would result. The bit error rate of the optimal detector for the single user system serves as a lower bound on the performance of any other detector. This single user bound is

$$
\mathrm{P}_{\mathrm{k}}(\mathrm{E})=\mathrm{Q}\left(\sqrt{\frac{\mathrm{w}^{\mathrm{k}}}{\mathrm{N}_{0}}}\right)
$$

where the Q-function

$$
Q(x)=\frac{1}{\sqrt{2 \pi}} \int_{x}^{\infty} \exp \left(\frac{-y^{2}}{2}\right) d y .
$$


The conventional DS/CDMA uses the same approach as the optimal receiver for the single user system. It detects the bit from user $\mathrm{k}$ by correlating the received signal with the chip sequence of user $\mathrm{k}$. Thus, the conventional detector makes its decision at the output of the matched filter bank:

$$
\hat{\mathbf{x}}^{\mathrm{k}}=\operatorname{sgn}(\mathrm{y} \mathbf{k})
$$

When MAI terms are significant (see (2-3)), the bit error rate of this detector is high. Note that MAI depends both on the cross-correlations and the powers of users. In the 2-user example above, if user 1 is much stronger than user 2 (the near-far problem), the MAI term $\mathrm{r} \mathrm{c}^{1} \mathrm{x}^{1}$ present in the signal of the second user is very large, and can significantly degrade performance of the conventional detector for that user. A multiuser detector called a successive interference canceller (decision-directed) can remedy this problem as follows. First, a decision $\hat{x}^{1}$ is made for the stronger user 1 using the conventional detector. Since user 2 is much weaker then user 1 , this decision is reliable from the point of view of user 2 . So, this decision can be used to subtract the estimate of MAI from the signal of the weaker user. The decision for user 2 is given by

$$
\hat{x}^{2}=\operatorname{sgn}\left(y^{2}-r c^{1} \hat{x}^{1}\right)=\operatorname{sgn}\left(c^{2} x^{2}+r c^{1}\left(x^{1}-\hat{x}^{1}\right)+z^{2}\right)
$$

Provided the decision of the first user is correct, all MAI can be subtracted from the signal of user 2 . The performance of this successive interference canceller for the weaker user is the same as the performance of the multistage detector proposed in [VA91]. (However, for the stronger user, the canceller can be more reliable.) If we fix the energy of the second user, and let the energy of the first user grow, the error rate of the successive interference canceller for the second user will approach the single-user bound. Thus, this detector is successful in combating the near-far problem. This simple example motivates the use of multiuser detectors for CDMA channels. Below, we will discuss several previously proposed multiuser detectors.

\subsection{The Decorrelating Detector}

As a step towards the most general formulation, consider the matrix version of the of the equivalent discrete time model (2-3). The output vector $y=\left[y^{1}, y^{2}, \ldots, y^{K}\right]^{T}$ (see (2-3)) can be expressed as

$$
\mathbf{y}=\mathbf{R} \mathbf{W} \mathbf{x}+\mathbf{z}
$$

where $\mathbf{R}$ and $\mathbf{W}$ are $\mathrm{K} \times \mathrm{K}$ matrices, and $\mathbf{z}$ is a colored Gaussian noise vector. The components of the matrix $\mathbf{R}$ are given by cross-correlations between signature waveforms

$$
\mathbf{R}_{k, j}=\int_{0}^{T} s_{k}(t) s_{j}(t) d t .
$$


The second matrix, $\mathbf{W}$, is diagonal with $\mathbf{W}_{\mathrm{k}, \mathrm{k}}$ given by the channel attenuation $\mathrm{c}^{\mathbf{k}}$ of the $\mathrm{k}$-th user. For example, in a two-user system, the matrix

$$
\mathbf{R}=\left(\begin{array}{cc}
1 & \mathbf{r} \\
\mathbf{r} & 1
\end{array}\right) \text {, }
$$

where $r$ is the cross-correlation between the signature waveforms of the users (2-9) (with $k=1, j=2$ ).

Inspection of (2-8) immediately suggests a method to solve for $\mathbf{x}$, whose components $x_{k}$ contain the bit information sought. If $\mathbf{z}$ was identically zero, we have a linear system of equations, $\mathbf{y}=\mathbf{R} \mathbf{W} \mathbf{x}$, the solution of which can be obtained by inverting $\mathbf{R}$ (it is invertible in most cases of interest [LU89]). With a non-zero noise vector $\mathbf{z}$, inverting $\mathbf{R}$ is still an effective procedure and actually optimal in certain circumstances, to be discussed later. This results in

$$
\tilde{\mathbf{y}}=\mathbf{R}^{-1} \mathbf{y}=\mathbf{W} \mathbf{x}+\tilde{\mathbf{z}}
$$

where it is seen that the information vector, $\mathbf{x}$, is recovered but contaminated by a new noise term (Figure $2-2)$. From (2-10), the signal of the $\mathrm{k}$-th user is

$$
\tilde{y}^{k}=c^{k} x^{k}+\tilde{z}^{k}
$$

The decision is $\hat{\mathbf{x}}^{\mathrm{k}}=\operatorname{sgn}\left(\tilde{\mathrm{y}}^{\mathrm{k}}\right)$.

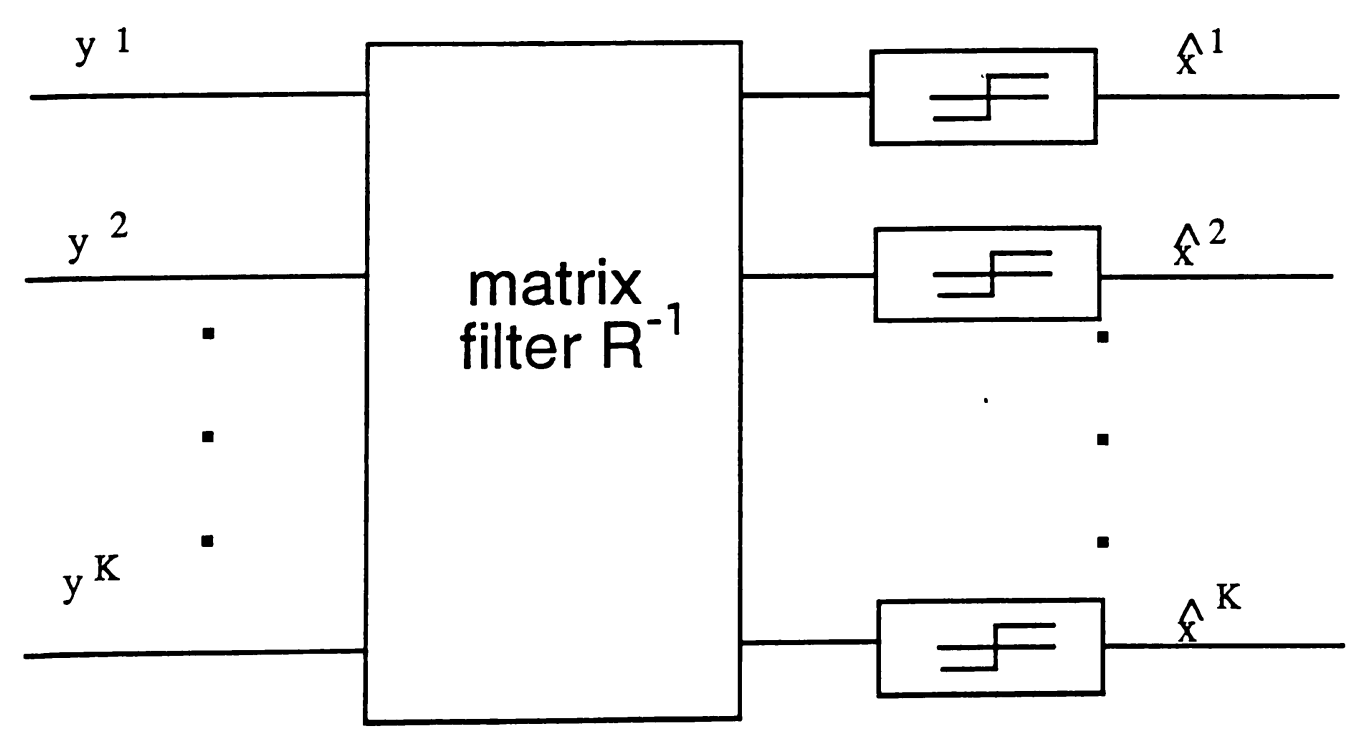

Figure 2-2. The Decorrelator for Synchronous CDMA

Note that the decorrelating detector completely eliminates MAI. However, the power of the noise $\tilde{z}^{\mathrm{k}}$ is $\mathrm{N}_{0}$ $\left(\mathbf{R}^{-1}\right)_{\mathbf{k}, \mathbf{k}}$ which is greater than the noise power $\mathrm{N}_{0}$ at the output of the matched filter (2-8). For example, 
for the 2-user system with the cross-correlation $r$, the noise power at the output of the decorrelating filter is $\mathrm{N}_{0} /\left(1-\mathrm{r}^{2}\right)$. The error rate of the decorrelator is given by

$$
P_{k}(E)=Q\left(\sqrt{\frac{w_{k}}{\left(N_{0} R^{-1} k, k\right)}}\right)
$$

The performance of the decorrelating detector degrades as the cross-correlations between users increase. The decorrelating detector also reduces to matrix inversion for the asynchronous case, although the derivation is more involved in that case [LU90]. The decorrelator has several desirable features. It does not require the knowledge of the users' powers, and its performance is independent of the powers of the interfering users. This can be seen from (2-11). Observe that neither signal nor noise terms depend on the powers of interferers. In addition, when users' energies are not known, and the objective is to optimize performance for the worst case MAI scenario, the decorrelator is the optimal approach [LU89]. In addition, the differentially coherent version of the decorrelator has been developed (see section 3 ). These properties of the decorrelator make it very well suited for the near-far environment.

Multiuser detection is closely related to equalization for intersymbol interference (ISI) channels [PR89]. For example, the decorrelating detector is analogous to the zero-forcing equalizer. Similarly, the MMSE linear multiuser detector [XI90] (also given by a matrix inverse) is the multidimensional version of the MMSE linear equalizer for the single-user ISI channel. The linear structure of these detectors often limits their performance. In the following section, we will describe several non-linear approaches to multiuser detection.

\subsection{The Optimal Detector}

The objective of maximum-likelihood sequence estimation (MLSE) is to find the input sequence which maximizes the conditional probability, or likelihood of the given output sequence [PR89]. For the simplified synchronous CDMA problem discussed above, the maximum likelihood decision for the vector of bits, $\mathbf{x}$, is given by

$$
\hat{\mathbf{x}}=\arg \left\{\max _{\mathbf{x} \in\{-1,+1\}^{\mathrm{x}}}\left[2 \mathbf{y}^{T} \mathbf{W} \mathbf{x}-\mathbf{b}^{T} \mathbf{W} \mathbf{R W b}\right]\right\}
$$

This equation dictates a search over the $2^{K}$ possible combinations of the components of the bit vector $\mathbf{x}$. For asynchronous CDMA, the MLSE detector can be implemented using the Viterbi algorithm [VE86]. The path metrics of this algorithm were derived by identifying the asynchronous CDMA channel with a single-user channel with periodically time-varying ISI. The memory of this equivalent channel is K-1 (the number of interferers), and therefore the resulting Viterbi algorithm has $2^{\mathrm{K}-1}$ states and requires $\mathrm{K}$ storage 
updates per transmission interval. Although the optimal detector has excellent performance, it is too complex for practical implementation, and we will not discuss it in greater detail.

\subsection{Non-Linear Suboptimal Multiuser Detectors}

In this section, we will consider several interference cancellation methods which utilize feedback to reduce MAI in the received signal. These algorithms can be broken into three classes:

(1) the multistage detectors [e.g., VA90,GI93,VA91,XI90];

(2) the decision-feedback detectors [DU92, DU93, DU94];

(3) successive interference cancellers (this idea is explicit or implicit in a number of papers)

Remark: $\quad$ Particular realizations of suboptimal multiuser detectors may use combinations of (1), (2), or (3).

The first two classes of algorithms are decision-directed. They utilize previously made decisions of other users to cancel interference present in the signal of the desired user. These algorithms require estimation of channel parameters and coherent detection. The algorithms in the third class use estimates of transmitted signals (e.g., outputs of the correlation receivers in [PA94]) to remove MAI components. They lend themselves to differentially coherent implementation. The algorithms of the second and third classes employ successive interference cancellation (also proposed in [VI90]), which requires ordering of users according to their powers. The signals of stronger users are demodulated first and cancelled from the signals of weaker users. This technique provides an efficient and practical solution to the near-far problem.

Several representatives from the three classes of non-linear detectors are described below.

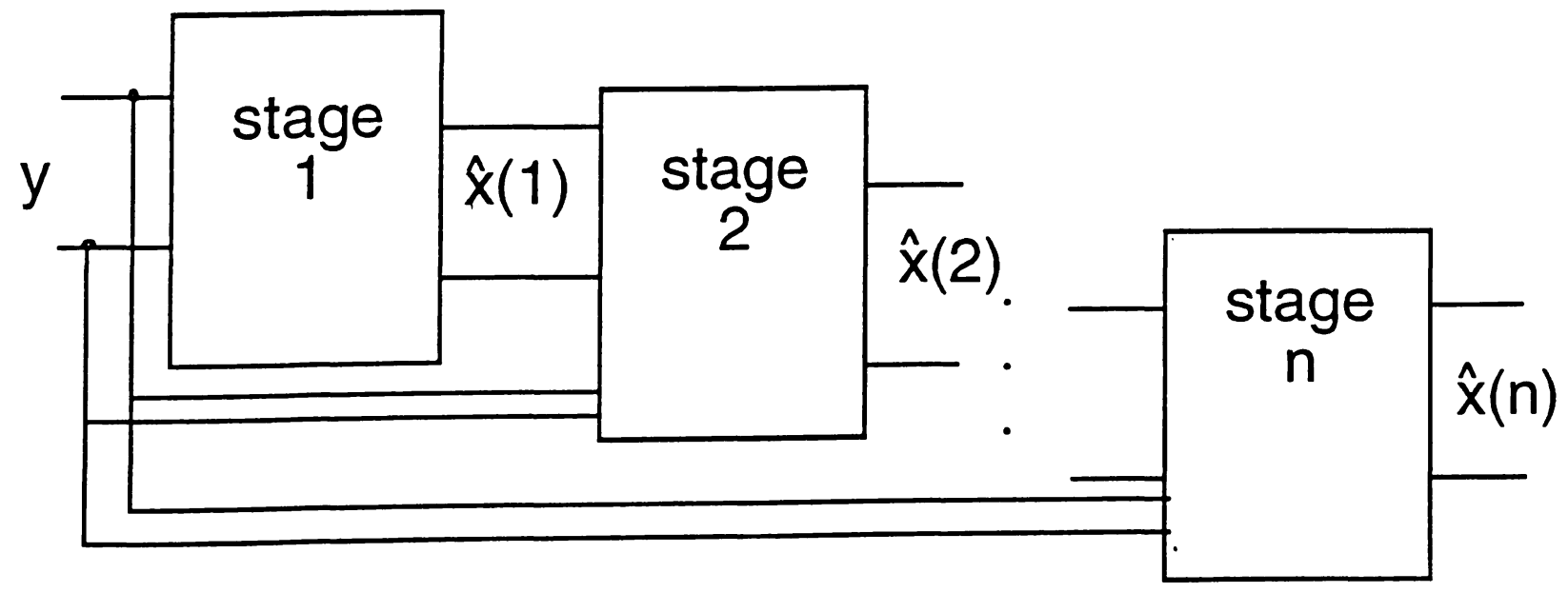

Figure 2-3. The Multistage Detector. 


\subsubsection{Multistage Detectors}

A diagram of the multistage detector proposed in [VA90, VA91] is shown in fig. 2-3. The n-th stage of this detector uses decisions of the (n-1)-st stage to cancel MAI present in the received signal. Due to delay constraints, it is desirable to limit the number of stages to two. For example, consider a two-stage detector with the conventional first stage for the synchronous 2 -user system with the cross-correlation $r(2-4)$. The decisions produced by the first stage (conventional) detector are $\hat{x}^{1}(1)$ and $\hat{x}^{2}(1)$ computed as in (2-6). The decisions of the second stage are $\hat{x}^{1}(2)=\operatorname{sgn}\left[y^{1}-r c^{2} \hat{x}^{2}(1)\right]$ and $\hat{x}^{2}(2)=\operatorname{sgn}\left[y{ }^{2}-r c^{1} \hat{x}^{1}(1)\right]$. The performance of this two-stage detector depends on the relative energies of the users. Clearly, if the first user is stronger than the second, the decisions of the second stage for user 2 agree with those of the decision-directed successive interference canceller described in the last paragraph of section 2.1. Thus, for the weaker user, the second stage produces more reliable decisions than the first stage. However, for the stronger user, feedback might not be beneficial since the decision produced by the conventional detector for the weaker user is poor. More reliable two-stage detector results if the conventional detector in the first stage is replaced by the decorrelator [VA91]. This example illustrates the issues which play a role the design of multistage detectors. In summary, the two important questions are:

- How to choose the initial stage.

- How to choose the subsequent stages of processing.

A discussion of different options for the initial and subsequent stages is given, along with further references, in [GI93].

\subsubsection{Decision-Feedback Detectors}

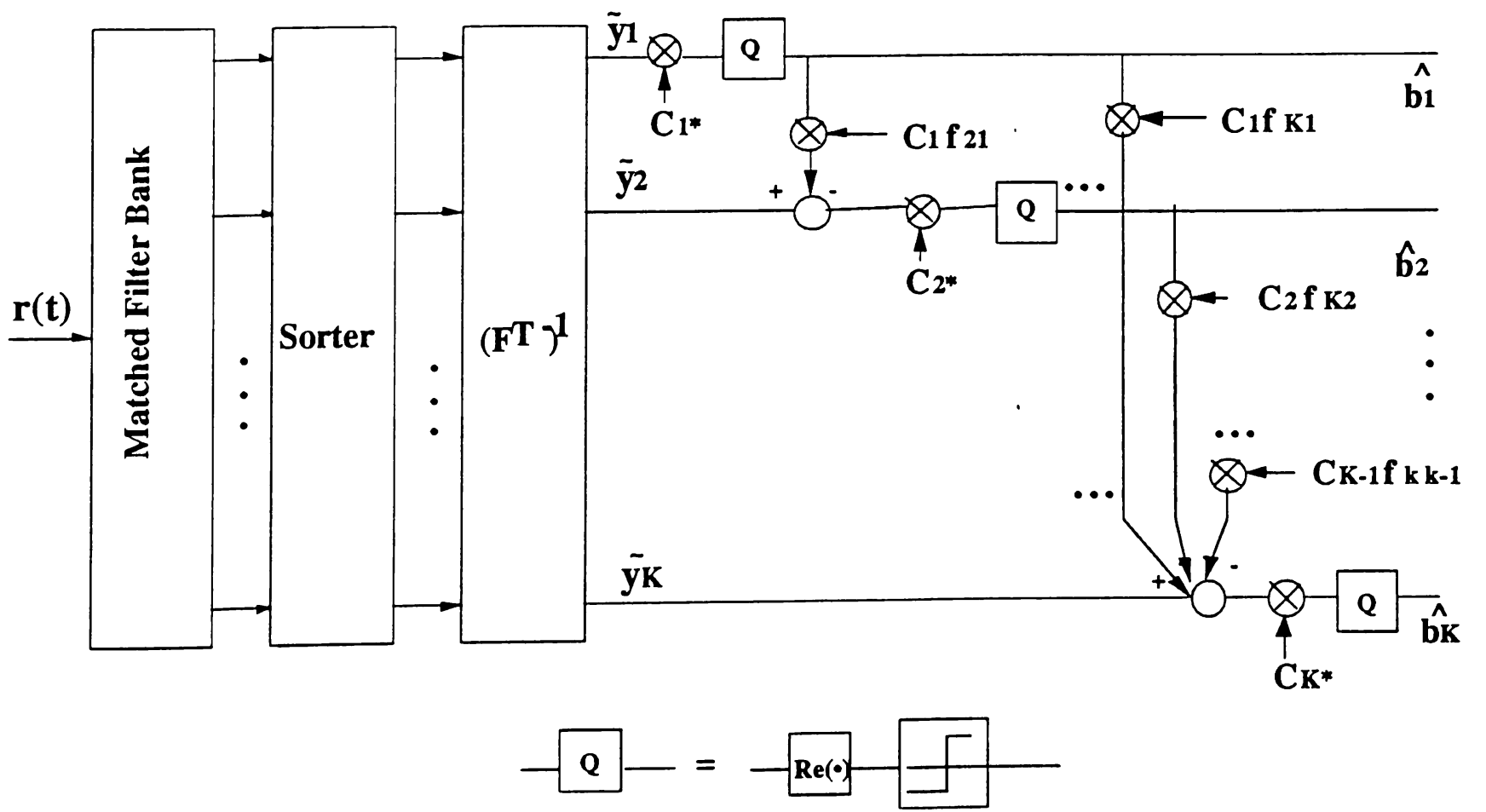

Figure 2-4. The decorrelating decision-feedback detector for synchronous CDMA 
The detectors proposed in [DU92, DU93, DU94] are multiuser decision-feedback equalizers, characterized by two matrix transformations: a forward filter and a feedback filter. These detectors are analogous to the decision-feedback equalizers employed in single user ISI channels [PR89]. However, in addition to equalization, the decision-feedback multiuser detectors employ successive cancellation. In each time frame, decisions are made in the order of decreasing user's strength, i.e. the stronger users make decisions first, allowing the weaker users to utilize these decisions. The sorting is performed by any multiuser detector with successive MAI cancellation. We will explain the rationale for using this particular order in section 24-3.

A diagram of the decorrelating (zero-forcing) decision-feedback detector for synchronous CDMA [DU93] is shown in Fig. 2-4. At the output of the sorter, users are ranked according to their powers, so that the strongest user is ranked first, and the weakest - last. Following the sorter, the noise whitening filter is applied. This filter is obtained by Cholesky factorization of the autocorrelation matrix, which yields a resulting MAI matrix which is lower triangular. Consequently, at the output of the whitening filter, the signal of the $\mathrm{k}$-th strongest user $\tilde{\mathrm{y}}_{\mathbf{k}}$ is given by :

$$
\tilde{y}_{\mathrm{k}}=\text { desired signal }+ \text { MAI due to stronger users }(1, \ldots, \mathrm{k}-1)+\text { noise. }
$$

In particular, the signal of the strongest user $\tilde{y}_{1}$ is not corrupted by MAI, and can be demodulated first. This decision is then used to subtract MAI from the signal of the second user, and so on. For the asynchronous CDMA, several decision-feedback detectors were derived in [DU92, DU94].

The performance of the decision-feedback detector is similar to that of the decorrelator for the strongest user, and gradually approaches the single user bound as user's power decreases relative to powers of interferers. Thus, for the decision-feedback detector, performance advantages with respect to the conventional or the decorrelating detectors are greater for relatively weaker users. This is also the case for multistage detectors with the decorrelating first stage. Figure 2-5 depicts typical performance of several detectors for the weakest user in a bandwidth efficient system. The signature waveforms for this asynchronous 4-user CDMA system were derived from Gold sequences of length 7 [DU94]. (see also [DU93, VA91].) In Figure 2-5, the conventional, decorrelating, decision-feedback and multistage detectors are compared for the weakest user (user 4). The powers of all users grow, but the differences between the powers remains the same. Note that the two-stage detector with the conventional first stage is interference-limited. Both the decision-feedback and the two-stage detector with the decorrelating first stage have excellent performance in this near-far scenario. 


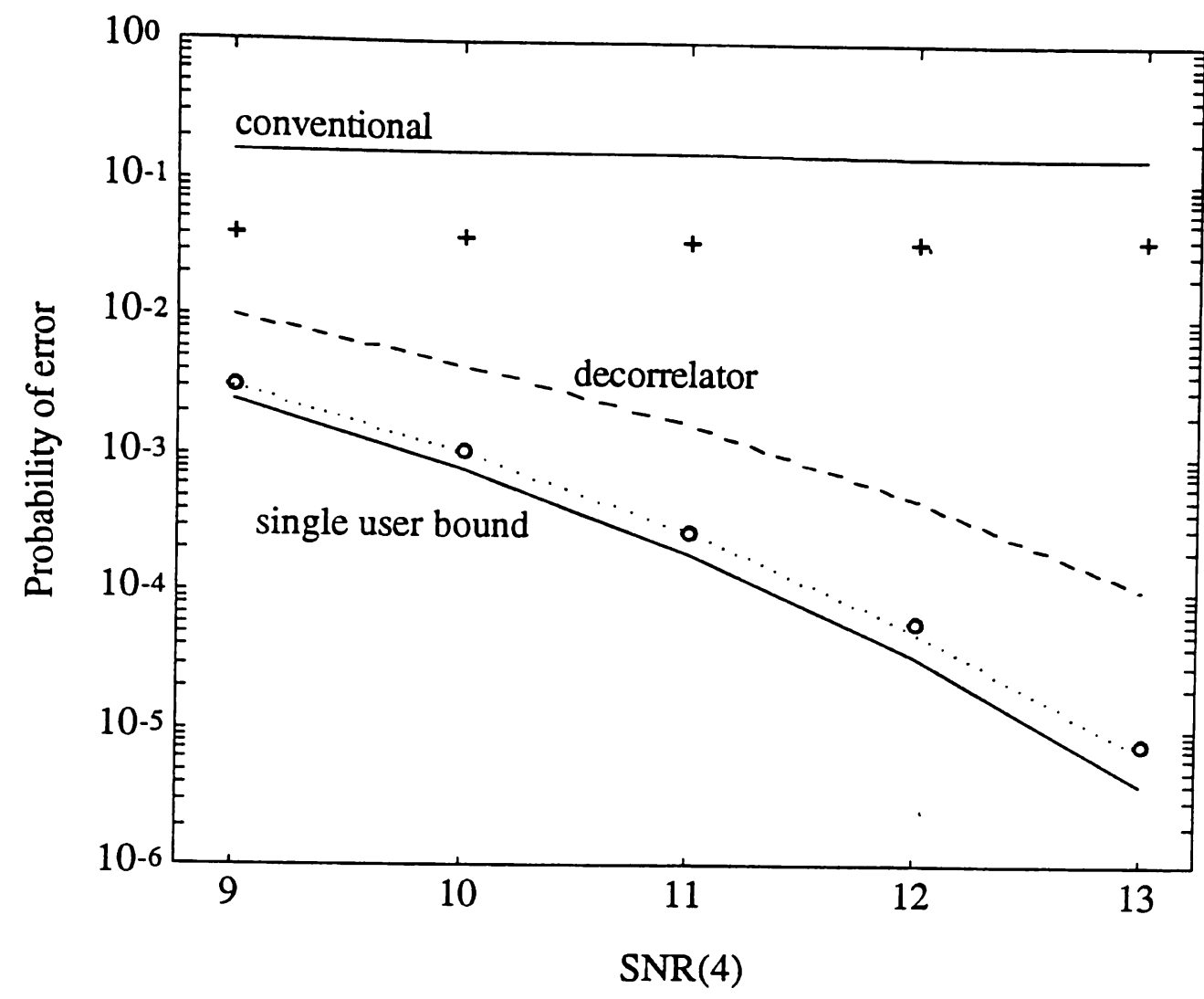

Figure 2-5. Error rates for user 4 in the four-user system of [DU94]. $\operatorname{SNR}(3)=\operatorname{SNR}(4)+3 \mathrm{~dB}, \operatorname{SNR}(2)=\operatorname{SNR}(4)+4 \mathrm{~dB}, \operatorname{SNR}(1)=\operatorname{SNR}(4)+5 \mathrm{~dB}$. $\begin{array}{ccc}\cdots & \text { df and ideal df } \\ + & + & \text { 2-stage df, } 1 \text { st stage - conventional } \\ 0 & 0 & 2 \text {-stage df, } 1 \text { st stage - decorrelator }\end{array}$

\subsubsection{Successive Interference Cancelers}

One approach to successive interference cancellation is to consider what would be the simplest augmentation to the conventional detector which would achieve some of the benefits of multiuser detection. This can be explained most simply by referring back to (2-3). In order to cancel the MAI, the factors $x^{j} c^{j}$ are needed, in addition to the cross-correlations. These can be obtained either with estimates of each of the factors $x^{j}$ and $c^{j}$ separately, i.e., separation of the bit estimate and power estimates. Alternately, one can estimate the product $x^{j} c^{j}$ directly by using the correlator output. We shall focus on the latter method because that requires the simplest augmentation to the conventional detector. It is found that using the correlator output to estimate $x^{j} c^{j}$ is sufficiently accurate to obtain improvement over the conventional detector.

As mentioned previously, it is most important to cancel the strongest signal before detection of the other signals because it has the most negative effect. Also, the best estimate of signal strength is from the strongest signal for the same reason that the best bit decision is made on that signal: the strongest signal 
has the minimum MAI since the strongest signal is excluded from its own MAI. This is the twofold rationale for doing successive cancellation in order of signal strength:

(i) Canceling the strongest signal has the most benefit.

(ii) Canceling the strongest signal is the most reliable cancellation.

In a number of studies (e.g., [DE92] ${ }^{* * *}$ [PA94], [PA94a]), it has been shown that this method of cancellation yields significant improvements over the conventional detector (but substantially less than the optimum multiuser detector).

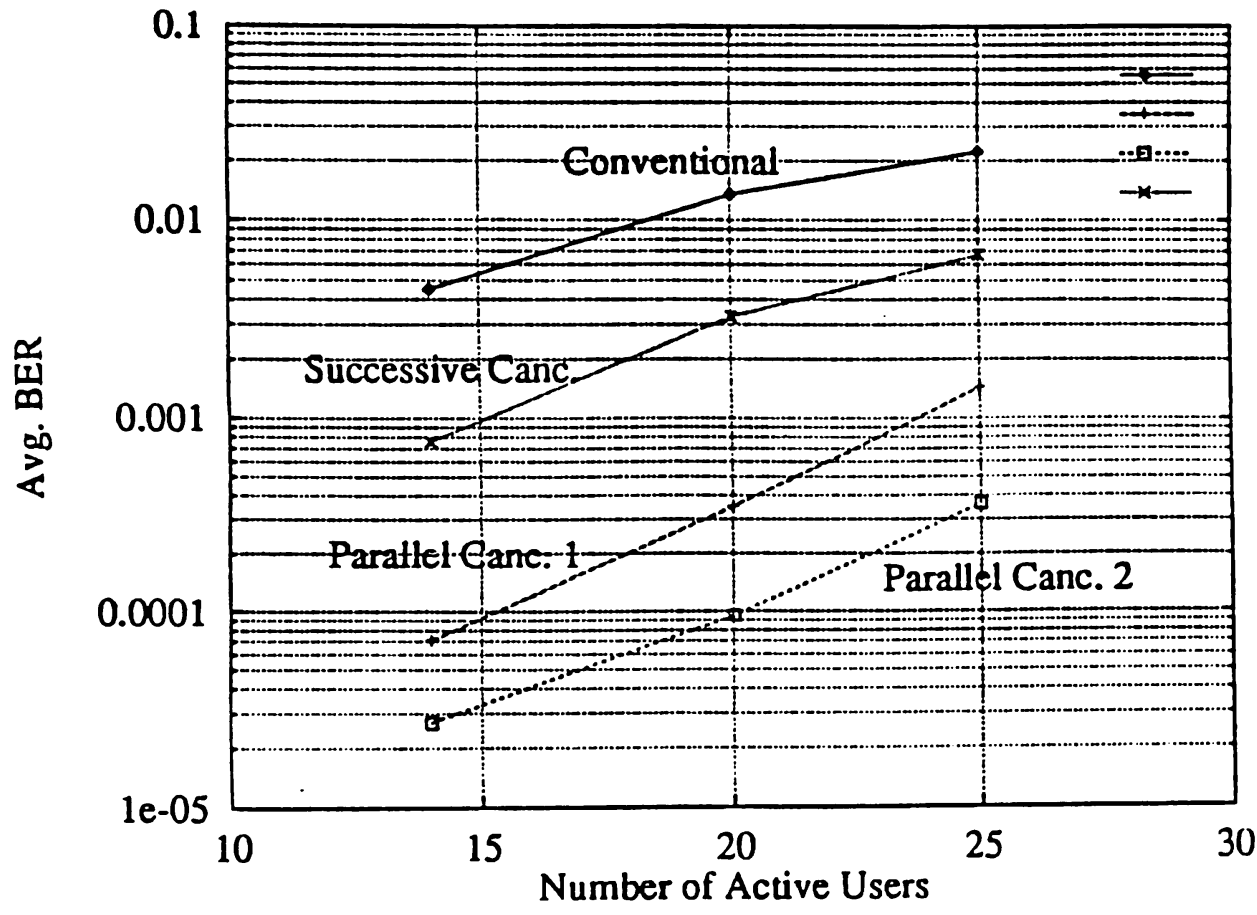

Figure 2.6 - BER vs \# of Active Users under Ideal Power Control (Asynchronous)

Successive cancellation works by successively subtracting off the strongest remaining signal. An alternative (the parallel method) is to simultaneously subtract off all of the users' signals from all of the others. It is found [PA94a] that when all of the users are received with equal strength, the parallel method outperforms the successive scheme (Figure 2.6). When the received signals are of distinctly different strengths (the more important case), the successive method is superior in performance (Figure 2.7). The important thing to note is that in both cases, both outperform the conventional detector and the unequal power case is the more important case.

*** [DE92] uses a method of cancellation in the spectral domain. 


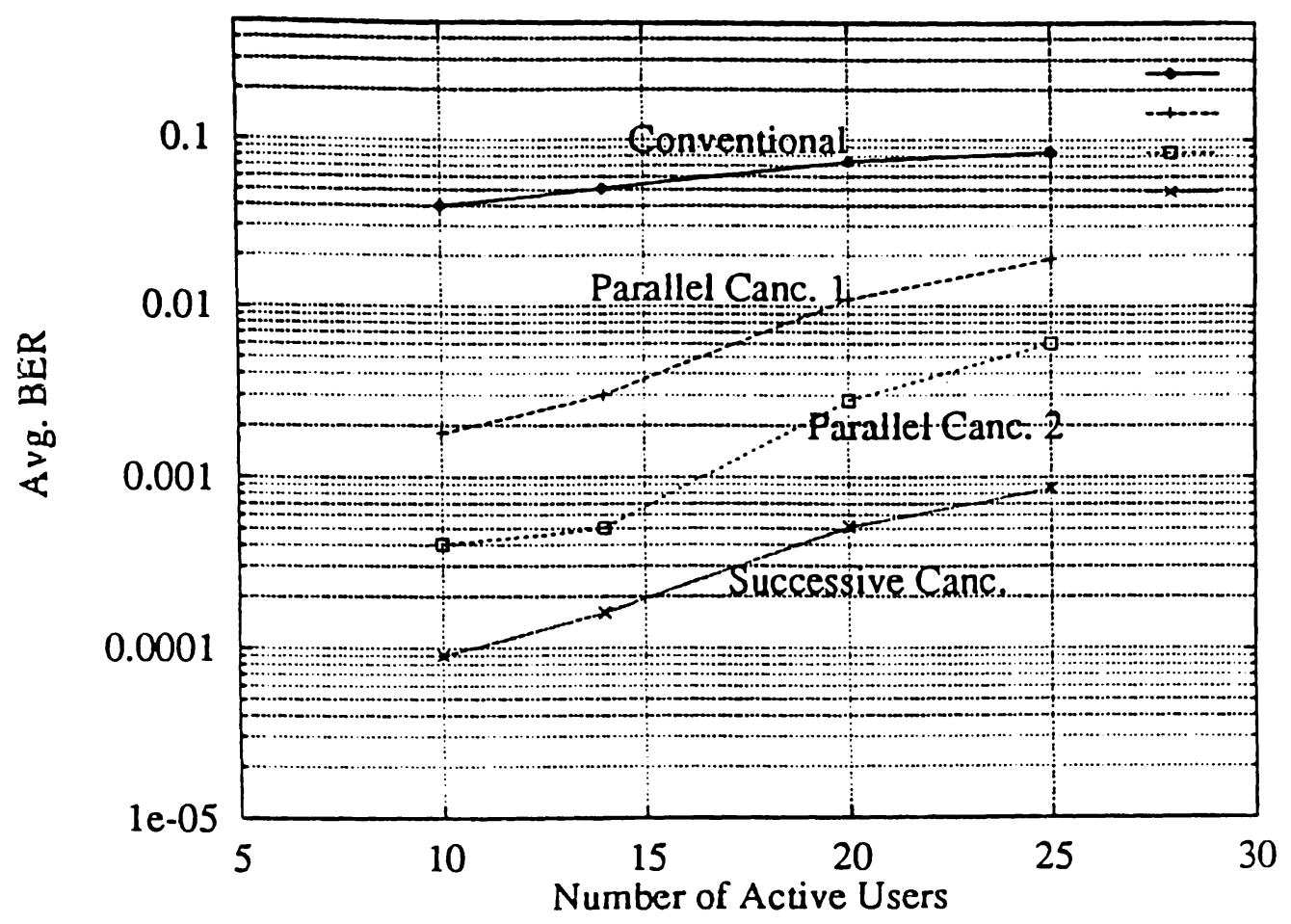

Figure 2.7 - BER vs \# of Active Users under Rayleigh Fading (Asynchronous)

The successive cancellation must operate fast enough to keep up with the bit rate and not introduce intolerable delay. For this reason, it will presumably be necessary to limit the number of cancellations. The ability to limit the number of cancellations is consistent with the objective of controlling complexity by choosing an appropriate performance/complexity tradeoff. For more information and references on successive interference cancellation, see [HO94].

\section{Noncoherent Multiuser Detection and Multipath Fading Channels}

For the sake of simplicity of exposition, most of the discussion in Section 2 concentrated on coherent multiuser reception. The underlying assumption there is that the multiuser receiver is able to estimate and track the phase of each active user in CDMA scenario. However, as stated earlier, a reverse link of a cellular CDMA system employs noncoherent reception since the pilot signal which provides a coherent reference is not available. We will briefly address the concept of noncoherent multiuser detection and also analyze the multiuser receiver performance in multipath fading channels.

All noncoherent multiuser techniques perform in-phase and quadrature $(I \& Q)$ demodulation before the detection. The noncoherent multiuser detection was first considered for differentially phase-shift keying (DPSK) [VA93]. The concept can be easily described as presented in Fig. 3.1. The decorrelating operation is performed both on in-phase and quadrature signal branches (after $I \& Q$ demodulation), so the phase information of the signal is preserved, although the phase is not being explicitely tracked. Since 
the decorrelating filter eliminates MAI, differentially coherent demodulation and the same decision logic can be applied as in single-user receiver. Moreover, this type of detector was shown to be optimally nearfar resistant.

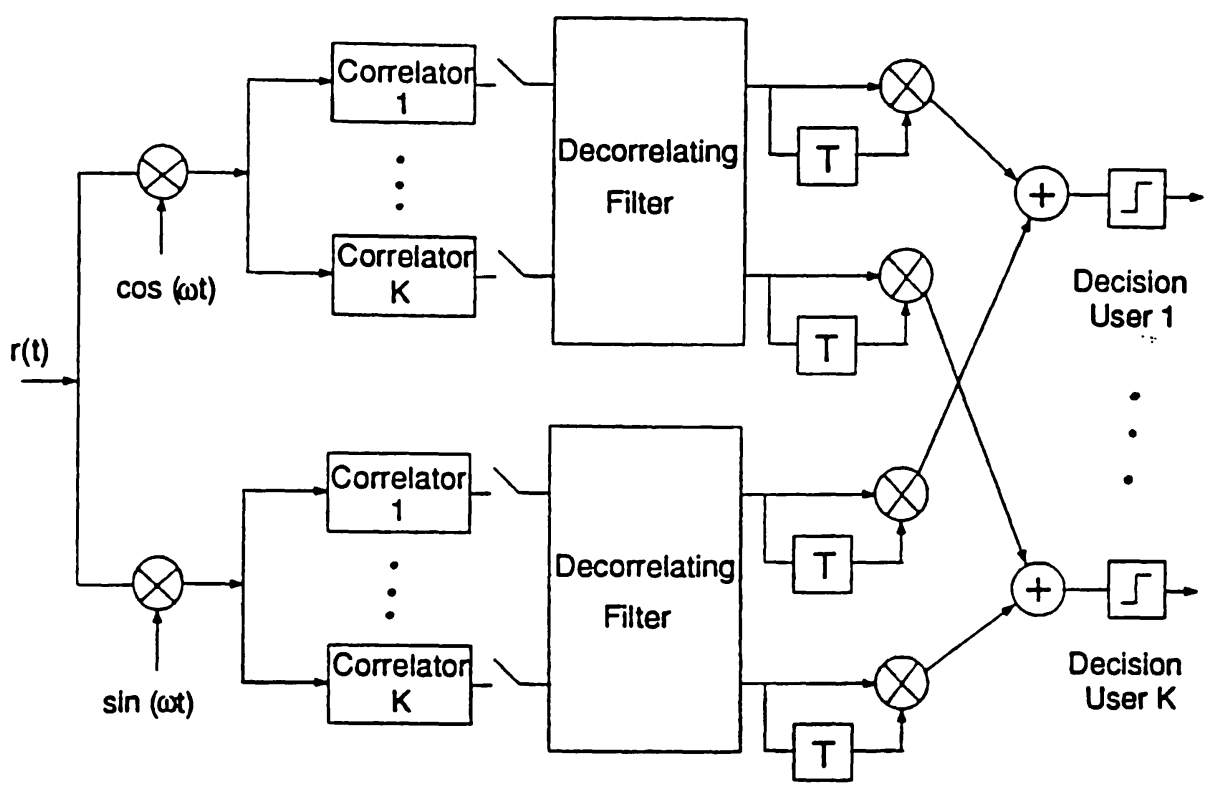

Figure 3.1 - Differentially coherent decorrelating multiuser detector.

The resulting expression for the error probability in a synchronous case, given by

$$
P_{k, D P S K}=\frac{1}{2} e^{-\frac{w_{k}}{N_{0}\left[\mathbf{R}^{-1}\right]_{k k}}}
$$

indicates that the performance loss compared to coherent reception is the same as in a single-user channel. The realization and performance of noncoherent decorrelating receiver do not depend on signal amplitudes and phases, and the only requirement for the described receiver to be near-far resistant is that chip sequences among the users are linearly independent.

Another noncoherent scheme is based on the idea of successive interference cancellation previously described in Section 2. A noncoherent version of successive interference cancellation uses a combination of M-ary orthogonal signaling and CDMA on the reverse link [PA94b]. After the $I \& Q$ demodulation at the receiver front-end, the received signal is correlated with respective $I \& Q$ chip sequences, the user's spreading code and with all M-ary symbols obtained from Walsh functions. The interference cancellation algorithm starts by decoding the strongest user first. The amplitude of the decoded user is estimated from the correlator output and the strongest user's signal is regenerated using this estimate and the 
corresponding chip sequence, and cancelled from the received signal. The cancellation is repeated until all users are decoded or until a limited number of cancellations are done. As in the case of differentially coherent multiuser reception, this concept can be extended to CDMA multipath fading channels as described in the next section.

\subsection{Multiuser Detection in Multipath Fading Channels}

As discussed in Section 1.2, multipath fading presents a major limitation to the performance of wireless CDMA systems. Examples include cellular mobile radio, indoor wireless communications, personal communication services and underwater acoustic local area networks. In these systems MAI is enhanced by multipath propagation, and near-far effects are produced not only by the difference in the distance between the transmitter and receiver but also by the fading on the propagation paths. While multipath propagation, usually encountered in an urban scenario, offers inherent diversity, certain scenarios in suburban areas result in a single-path propagation [TU84], [LE91]. This depends on whether the individual multipaths can be resolved which in turn depends on whether the chip duration is small enough relative to the separation between multipaths. In the case of single-path propagation small but nonzero cross-correlations among chip sequences can cause severe near-far problem in the presence of fading. When there is only a single fading path for each active user and interference is relatively strong, there are no means of diversity to overcome fading of the desired signal below the level of MAI, unless the explicit diversity using distributed antennas is introduced. Since multiuser receivers are near-far resistant, they significantly improve the CDMA system performance in the single-path scenario [ZV94], [DU93a], [PA94b], [WU94].

When the chip duration is small enough to resolve the different multipath receptions, multipath diversity is exploited to improve the performance in the presence of MAI. The conventional receiver in the case of multipath fading channel consists of a bank of RAKE receivers, one for each active user, at the base station and one for the desired user in a mobile. A summary of the research efforts on conventional reception techniques is given in [KC93]. Consequently, having in mind the multipath combining property of RAKE receiver, all multiuser techniques in multipath fading channels utilize some form of RAKE structure at the receiver front-end. With multipath resolution available, multiuser detection and multipath diversity reception are combined to provide the reliable receiver performance.

The optimal MLSE receiver for multipath fading CDMA channel consists of the same front-end of RAKE filters, followeed by a dynamic programming algorithm of the Viterbi type. This optimal structure provides the same order of diversity and asymptotically has the same error probability as RAKE receiver in the single-user case [ZV92], at the expense of high complexity.

Both linear and nonlinear suboptimal noncoherent multiuser receivers for multipath fading channels have been proposed. A RAKE receiver can be interpreted as a combiner of the correlators outputs, where the 
combining method depends on the modulation type. To eliminate the effects of MAI prior to the combining process, linear multipath decorrelating receivers [ZV92a], [WI93] perform the decorrelating operation on $K L$ correlator outputs, where $L$ is the number of resolvable fading paths and $K$ is the number of active users as depicted in Fig. 3.2. Consequently, the equal-gain diversity combining for DPSK signaling is performed on signals which suffer from less interference. The performance loss due the noise enhancement in the presence of other active users is modest, for the typical mobile radio scenarios it is on the order of $3 d B$ compared to single-user RAKE performance over the whole range of SNRs [ZV93]. Similar performance degradations are observed for the coherent linear multiuser receivers [KL93]. When a fraction of the multipath power is captured due to limited number of RAKE correlators, only a portion of MAI is eliminated by decorrelating operation and residual MAI may cause the performance degradation (recall Section 1). In that case additional antenna diversity was shown to be effective in reducing the effects of the residual MAI [ZV93]. Another combination of multiuser detection and antenna diversity is given in [KO90].

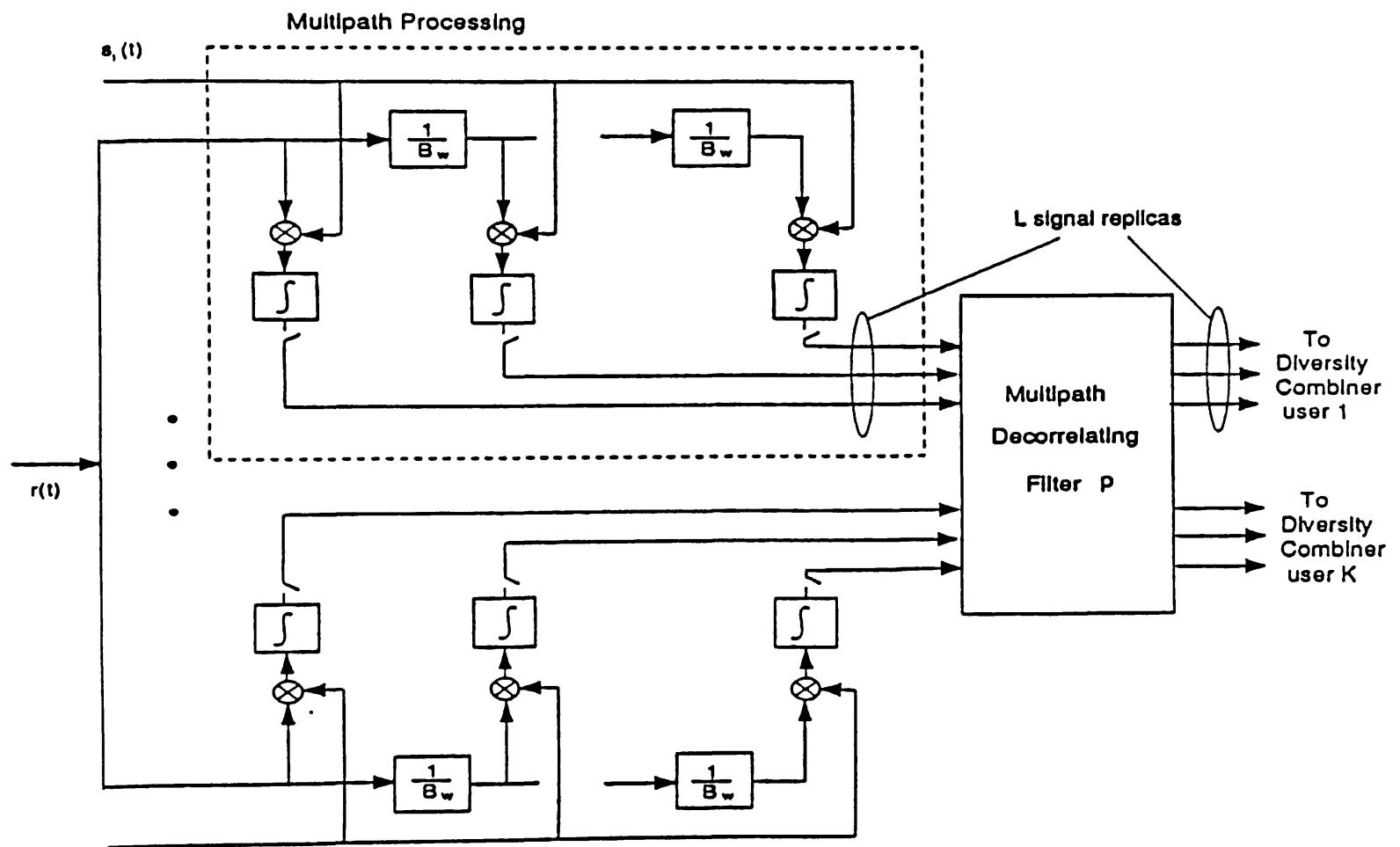

\section{Figure 3.2 - Differentially coherent multipath decorrelating/multipath combining linear
multiuser receiver.}

Nonlinear techniques for multipath fading channels rely on interference cancellation and inherently employ the regeneration of the interfering signals. The major difference among numerous interference cancellation techniques is in the methods for the channel parameters estimation and interfering signal reconstruction. A successive interference cancellation receiver for noncoherent $\mathrm{M}$-ary orthogonal modulation which uses the outputs of the correlators to estimate the signal amplitudes, and hence does not require any separate 
channel estimates is given in [PA94]. Since RAKE receivers are used in a front-end, each multipath arrival tracked by RAKE is cancelled from the received signal using the appropriate correlator output as shown in Fig. 3.3.

For any of the multipath multiuser detectors, the residual MAI problem of Section 1.5 could arise when the number of cancelled paths is smaller than the total number of multipaths in the channel.

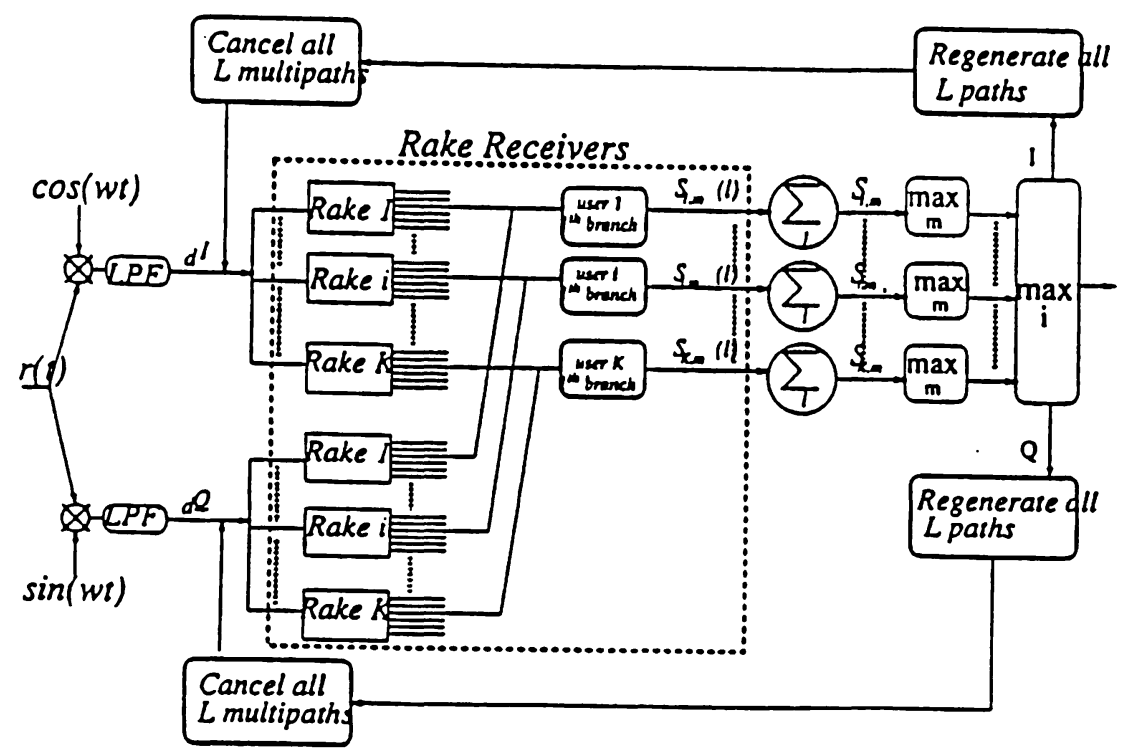

Figure 3.3 - Noncoherent receiver with interference cancellation and multipath combining.

\section{Issues in Practical Implementations}

\subsection{Complexity}

Major obstacles to the application of the multiuser detectors in practical wireless systems are processing complexity and possible processing delay. The optimal multiuser MLSE receiver is clearly too complex for any application in a system with a large user population, and most of the efforts are focused on implementation of suboptimal structures. Two promising techniques regarding receiver complexity are the linear sliding window decorrelating receiver [WI93] and the successive interference canceller using correlator outputs for amplitude estimates already discussed.

The sliding window decorrelating algorithm allows what may be practical implementation of the decorrelating detector, both for infinite and finite data block lengths in asynchronous CDMA systems, by solving the linear system of equations described in [WI93]. The major cost in each iteration is due to recomputation of the linear system due to change in relative delays among users, dynamic selection of 
multipath and voice activity exploration. The computationally efficient algorithm for updating the coefficients of the decorrelating filter is proposed by exploiting the parallelism in the linear system solution [WI93a]. The number of operations for the correction of the matrix filter coefficients is on the order of $K \mathrm{x} N$ where $K$ is the number of active users in CDMA system and $N$ is the length of the sliding window. The readily available technology for high-speed zero-forcing equalizers makes the decorrelator one of the simplest interference cancellation technique to implement [WI93a].

The successive interference cancellation scheme of Section 2.4.3 may be the simplest augmentation to the conventional detector. The major complexity in the multipath environment comes from tracking each multipath at the RAKE receiver, which involves for each path $M$ coefficients corresponding to the possible $M$ orthogonal symbols [PA94]. These coefficients are generated by a Walsh-Hadamard Transform (WHT) and the speed of performing the transform determines the processing delay of the canceller. To ensure the real-time implementation the speed of WHT must be at least $I \mathrm{x} R$ where $I$ is the number of possible cancellations and $R$ is the targeted symbol rate in the CDMA system. The complexity of the successive interference cancellation scheme can be controlled by limiting the number of cancellations. That is, one can achieve a compromise between performance and complexity. Observe that there is decreasing improvement with subsequent cancellations because cancellations are done in order of strength of the different users' signals.

\subsection{Sensitivity and Robustness}

Almost all of the discussions and analyses of multiuser detection have assumed a number of idealizations. For example, in cancelling out a chip sequence, it has been assumed that there is a perfect synchronization of each user. Clearly, if there are tracking errors, the chip sequences being cancelled will be offset and imperfect cancellation will be performed. The pertinent question is whether the tracking error tolerable for the conventional detector is tolerable for the cancellation receiver, or how much tighter it must be for multiuser detection. For the conventional detector, the synchronization must be within a fraction of a chip duration. The impact of tracking errors on the performance of successive interference cancellation receiver is analyzed in [CH94]. For numerical results presented here, the interference cancellation scheme was subject to pessimistic conditions:

- Did not use averaging of the correlator outputs for amplitude estimates which significantly improves cancellation performance.

- Assumed equal received powers (perfect power control). The improvement over the conventional detector is much greater in the more realistic case of unequal received powers.

Figure 4.1 shows results for a processing gain (number of chips/bit) $N=31$ and the total number of users is varied from 5 to 20 . There are 3 curves each for the interference cancellation scheme and the conventional detector. Each curve represents different standard deviation $e$ of tracking error normalized 
with respect to chip duration ( $e=0$ is zero tracking error). The interference cancellation scheme retains superiority over the conventional detector. Similar types of results were found in [LE94] from simulation of the scheme of [DE92]. While it is premature to draw any general conclusions about robustness of the interference cancellation receivers at this point, these results are promising.

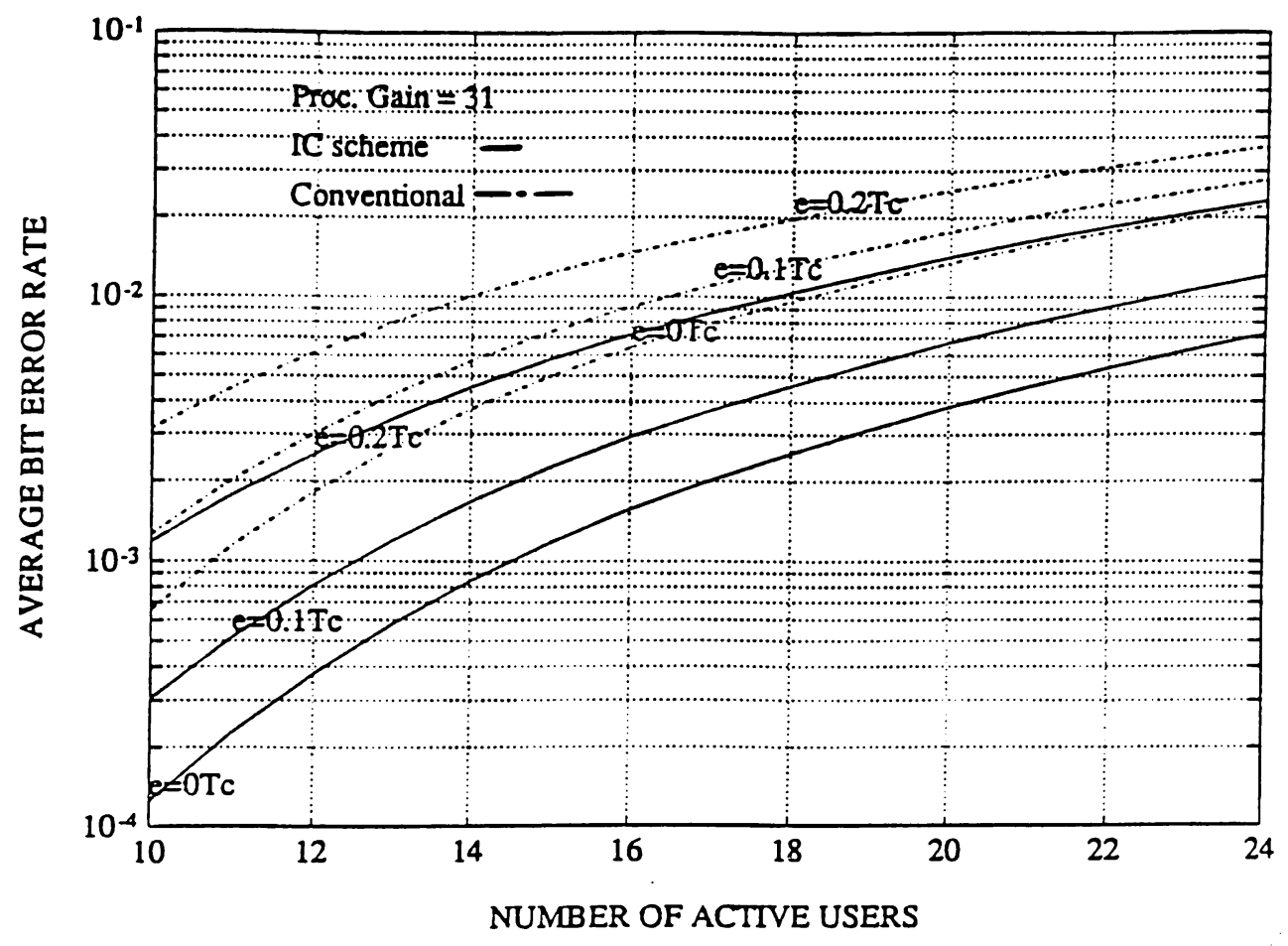

Figure 4.1.- BER Performance Degradation with Tracking Errors

\section{Concluding Remarks}

The theoretical bases of optimal multiuser detection are well understood. Given the prohibitive complexity of optimum multiuser detectors, attention has been focused on suboptimal detectors, and properties of these detectors are well understood by now. The next stages of investigation, involving implementation and robustness issues, are accelerating now and will lead to determination of the practical and economic feasibility of the multiuser detector. Initial studies of robustness show that robustness does not appear to be a fatal flaw. Further investigations into practicality will include actual hardware implementations.

\section{$\underline{\text { References }}$}

[CA87] James W. Carlin, Yeheskel Bar-Ness, Stephen Gross, Michael I. Steinberger and William E. Studdiford, "An IF Cross-Pol Canceller for Microwave Radio Systems," IEEE Journal on Selected Areas in Communications, Vol. SAC-5, No. 3, pp.502-514, April 1987.

[CH92] L.F. Chang, "Dispersive Fading Effects in CDMA Radio Systems," Proc. of ICUPC '92 Dallas, TX, September 1992, pp. 185-189. 
[CH94] Fang C. Cheng and Jack M. Holtzman, "Effect of Tracking Errors on DS/CDMA Successive Interference Cancellation," To appear in Proc. of Third Communication Theory Mini Conference in Conjunction with Globecom '94 , San Francisco, CA, November 1994.

[DE92] P. Dent, B. Gudmundson and M. Ewerbring, "CDMA-IC: A Novel Code Division Multiple Access Scheme Based on Interference Cancellation," Proc. of PIMRC '92, Boston, MA, October 1992, pp. 98-102.

[DU92] A. Duel-Hallen, "On Suboptimal Detection for Asynchronous Code-Division Multiple Access Channels," Proc. of the Twenty-Sixth Annual Conference on Information Sciences and Systems, Princeton University, Princeton, NJ, March 1992, pp. 838-843.

[DU93] A. Duel-Hallen, "Decorrelating Decision-Feedback Multiuser Detector for Synchronous Code-Division Multiple Access Channel," IEEE Transactions on Communications, Vol. COM-41, No.2, pp. 285-290, February 1993.

[DU93a] A. Duel-Hallen, "Performance of Multiuser Zero-Forcing and MMSE Decision Feedback Detectors for CDMA Channels," Conference Record of the Second Communication Theory Mini-Conference in conjunction with Globecom '93, Houston, TX, December 1993, pp. 82-86.

[DU94] A. Duel-Hallen, "A Family of Multiuser Decision-Feedback Detectors for Asynchronous Code-Division Multiple Access Channels," IEEE Transactions on Communications, to appear.

[GI91] K.S. Gilhousen, I.M. Jacobs, R. Padovani, A.J.Viterbi, L.A. Weaver,Jr. and C.E. Wheatley III, "On the capacity of a Cellular CDMA System," IEEE Transactions on Vehicular Technology, Vol. VT-40, No. 2, pp. 303-312, May 1991.

[GI93] T. R. Giallorenzi and S. G. Wilson, "Decision Feedback Multiuser Receivers for Asynchronous CDMA Systems," Proc. of Globecom'93, Houston, TX, Nov.- Dec. 1993, pp. 1677-1681.

[HO94] J. M. Holtzman, "DS/CDMA Successive Interference Cancellation," Proc. of ISSSTA '94 , Oulu, Finland, July 1994.

[KC93] C. Kchao and G. Stuber, "Performance Analysis of a Single Cell Direct Sequence Mobile Radio System," IEEE Transactions on Communications, Vol. COM-41, No. 10, pp. 15071516, October 1993.

[KL93]

A. Klein and P. Baier, "Linear Unbiased Data Estimation in Mobile Radio System Applying CDMA,". IEEE Journal of Selected Areas in Communications, Vol. SAC-11, No. 7, pp. 1058-1066, September 1993.

[KO83] R. Kohno, M. Hatori and H. Imai, "Cancellation Techniques of Co-Channel Interference in Asynchronous Spread Spectrum Multiple Access Systems," Electronics and Communications, Vol. 66-A, No. 5, pp. 20-29, 1983.

[KO90] R. Kohno, H. Imai, M. Hatori and S. Pasupathy, "Combination of an Adaptive Array Antenna and a Canceller of Interference for Direct-Sequence Spread- Spectrum MultipleAccess System," IEEE Journal on Selectd Areas in Communications, Vol. SAC-8, No.4, pp. 675-682, May 1990.

[LE91]

W. Lee, "Overview of Cellular CDMA," IEEE Transaction on Vehicular Technology, Vol. VT-40, No. 2, pp. 291-302, May 1991. 
[LE94] L.Levi, F. Muratore and G. Romano, "Simulation Results for a CDMA Interference Cancellation Technique in a Rayleigh Fading Channel," Proc. of 1994 International Zurich Seminar on Digital Communications, Zurich, Switzerland, March 1994, pp.

[LI83] J. S. Lim, Ed., Speech Enhancement, Prentice-Hall, Englewood Cliffs, NJ, 1983.

[LU89] R. Lupas and S. Verdu, "Linear Multiuser Detectors for Synchronous Code-Division Multiple-Access Channel," IEEE Transactions on Information Theory, Vol. IT-35, No. 1, , pp. 123-136, January 1989.

[LU90] R. Lupas and S. Verdu, "Near-Far Resistance of Multiuser Detectors in Asynchronous Channel," IEEE Transactions on Communications, Vol. COM-38, No. 4, pp. 496-508 April 1990.

[PI91] R.L. Pickholtz, L.B. Milstein and D.L.Schilling, "Spread Spectrum for Mobile Communications" IEEE Transactions on Vehicular Technology, Vol. VT-40, No. 2, pp. 313-322, May 1991.

[PA94] P. Patel and J. Holtzman, "Analysis of a Simple Successive Interference Cancellation Scheme in DS/CDMA System," to appear in JSAC - Special Issue on CDMA, First Quarter 1994.

[PA94a] P. Patel, and J. Holtzman, "Performance Comparison of a DS/CDMA System using a Successive Interference Cancellation (IC) Scheme and a Parallel IC Scheme under Fading," Proc. of ICC'94, New Orleans, LA , May 1994, pp. 510-515.

[PA94b] P.R. Patel and J.M. Holtzman, "Analysis of Successive Interference Cancellation in M-ary Orthogonal DS-CDMA System with Single Path Rayleigh Fading," Proc. of 1994 International Zurich Seminar on Digital Communications, Zurich, Switzerland, March 1994, pp. $150-161$.

[PR89] John G. Proakis, "Digital Communications," McGraw-Hill, 1989.

[SA85] J. Salz, "Digital Transmission Over Cross-Coupled Linear Channels," AT\&T Tech, Journal, Vol. 64, No. 6, pp. 1147-..., July-Aug. 1985.

[SC79] K. S. Schneider, "Optimum Detection of Code Division Signals," IEEE Trans. on Aerospace and Electronic Systems, Vol. AES-15, No. 1, p. 181-185, Jan. 1979.

[SI85]

[TU84]

M.K. Simon, J.K. Omura, R.A. Scholtz and B.K. Levitt, "Spread Spectrum Communications, Vols. I-III, Computer Science Press, Rockville, MD 1985.

G. Turin, "The Effects of Multipath and Fading on the Performance of Direct Sequence CDMA Systems," IEEE Journal on Selected Areas in Communications, Vol. SAC-2, No.4, pp 597-603, July 1984.

[VA90] M. K. Varanasi and B. Aazhang, "Multistage Detection in Asynchronous Code Division Multiple-Access Communications," IEEE Transactions on Communications., Vol. COM38, No. 4, pp. 509-519, April 1990.

[VA91]

M. K. Varanasi and B. Aazhang, "Near-Optimum Detection in Synchronous Code-Division Multiple Access Systems," IEEE Transactions on Communications, Vol. COM-39, pp. 725-736, May 1991.

[VA93] M. Varanasi, "Noncoherent Detection in Asynchronous Multiuser Channels," IEEE Transactions on Information Theory, Vol. IT-39, No.1, pp. 157-176, January 1993. 
S. Verdu, "Minimum Probability of Error for Asynchronous Gaussian Multiple Access Channels," IEEE Trans. on Info. Theory, Vol. IT-32, No. 1, January 1986.

[VE86a] S. Verdu, "Optimum Multiuser Asymptotic Efficiency," IEEE Transactions on Communications, Vol. Com-34, No. 9, pp. 890-897, September 1986.

[VE94] S. Verdu, "Adaptive Multiuser Detection," Proc. of ISSSTA '94, Oulu, Finland, July 1994.

[VI90] A. Viterbi, "Very Low Rate Convolutional Codes for Maximum Theoretical Performance of Spread-Spectrum Multiple-Access Channels," IEEE Journal on Selected Areas in Communications, Vol. SAC-8, No. 4, pp. 641-649, May 1990.

[VI94] A.J. Viterbi, "The Orthogonal-Random Wave form Dichotomy for Digital Mobile Personal Communications," IEEE Personal Communications, pp. 18-24, First Quarter 1994.

[WI85] B. Widrow and Samuel D. Stearns, Adaptive Signal Processing, Prentice-Hall, Englewood Cliffs, NJ, 1985.

[WI93] S. Wijayasuriya, J. McGeehan and G. Norton, "RAKE Decorrelating Receiver for DSCDMA Mobile Radio Networks," Electronic Letters, Vol. 29, No. 4, pp. 395-396, February 1993.

[WI93a] S. Wijayasuriya, G. Norton and J. McGeehan, "A Novel Algorithm for Dynamic Updating of Decorrelator Coefficients in Mobile DS-CDMA," Proc. of the 4th International Symposium on Personal, Indoor and Mobile Radio Communications, Yokohama, Japan, October 1993, pp. 292-296.

[WU94] H.Y. Wu and A. Duel-Hallen, "Performance of Multiuser Decision-Feedback Detectors for Flat Fading Synchronous CDMA Channels," Proc. of the 28th Annual Conference on Information Sciences and Systems, Princeton University, Princeton, NJ, March 1994.

[XI90] Z. Xie, R. T. Short and C. K. Rushforth, "A Family of Suboptimum Detectors for Coherent Multiuser Communications," IEEE Journal on Selected Areas in Communications, Vol. SAC-8, No. 4, pp. 683-690, May 1990

[ZV92] Z. Zvonar and D. Brady, "Optimum Detection in Asynchronous Multiple-Access Multipath Rayleigh Fading," Proc. of the Twenty Sixth Annual Conference on Information Sciences and Systems , Princeton University, March 1992, pp. 826-831.

[ZV92a] Z. Zvonar and D. Brady, "Coherent and Differentially Coherent Multiuser Detectors for Asynchronous CDMA Frequency-Selective Channels," Proc. MILCOM '92, San Diego, CA, October 1992, pp 442-446.

[ZV93] Z. Zvonar, "Multiuser Detection and Diversity Combining for Wireless CDMA Systems," Proceedings of the Fourth WINLAB Workshop on Third Generation Wireless Information Networks, WINLAB Wireless Information Network Laboratory, Rutgers University, NJ, October 1993, pp. 65-89.

[ZV94] Z. Zvonar and D. Brady, "Multiuser on in Single-Path Rayleigh Fading Channels", IEEE Transactions on Communications. ,Vol. COM-42, No.4, pp.1729-1739, April 1994. 